ISSN 0103-5150

Fisioter. Mov., Curitiba, v. 25, n. 2, p. 453-459, abr./jun. 2012 Licenciado sob uma Licença Creative Commons

\title{
0 uso do laser na reabilitação das desordens temporomandibulares
}

\section{The use of laser in the rehabilitation of temporomandibular disorders}

\author{
Thiago de Oliveira Assis ${ }^{[a]}$, Matheus dos Santos Soares ${ }^{[b]}$, Márcio Melo Victor ${ }^{[c]}$ \\ [a] Mestre em Patologia, docente da Universidade Federal da Paraíba, João Pessoa, PB - Brasil, e-mail: \\ thiago.oa@hotmail.com \\ [b] Graduado em Fisioterapia pela Faculdade de Ciências Médicas, Campina Grande, PB - Brasil, e-mail: \\ matheus_ssoares@hotmail.com \\ [c] Graduado em Fisioterapia pela Faculdade de Ciências Médicas, Campina Grande, PB - Brasil, e-mail: \\ marciomelovictor@hotmail.com
}

\section{Resumo}

Introdução: Entende-se disfunção temporomandibular comoum termo coletivo que envolve um grande número de problemas clínicos que afetam os músculos mastigatórios, a articulação temporomandibular (ATM) e estruturas associadas. Dentre os principais sinais e sintomas, incluem-se dores nos músculos da mastigação ou na ATM, ruídos articulares, limitação de abertura, retração gengival, oclusão inadequada, distúrbios auditivos, cefaleias, sensibilidade em toda a musculatura do sistema estomatognático e cervical. Objetivos: Estudar a influência da laserterapia de baixa intensidade (diodo) no tratamento das desordens temporomandibulares. Materiais e métodos: Trata-se de uma revisão sistemática. Foram consultadas as seguintes bases de dados: BIREME, MEDLINE, PubMed, Science Direct e LILACS. Os termos de busca utilizados foram "temporomandibular laser therapy" e "ATM laser treatment", em inglês e português. Não foram estabelecidos limites de busca. Após a busca independente de dois revisores, foram encontrados 1.344 artigos e, após a aplicação dos critérios de exclusão, 12 artigos foram selecionados para a análise. Resultados: Dos 12 artigos selecionados, apenas em 1 (um) deles não foi verificada qualquer melhora do quadro doloroso na ATM em virtude dos diversos tipos de DTM. Conclusão: Apesar dos resultados satisfatórios do uso do laser na redução sintomática da dor nos DTM, ainda é controversa a utilização dos seus parâmetros para cada caso. Por isso, sugerimos a realização de outros estudos para que essas lacunas sejam preenchidas com o objetivo 
maior de promover uma melhor qualidade de vida para as pessoas que sofrem quaisquer transtornos na região da ATM.

Palavras-chave: Lasers semicondutores. Transtornos da articulação Temporomandibular.

Modalidades de fisioterapia.

\section{Abstract}

Introduction: It is understood by temporomandibular dysfunction as a collective term that involves a large number of clinical problems affecting the masticatory muscles, the temporomandibular joint (TMJ) and associated structures. Among the major signs and symptoms there are pain in the masticatory muscles or TMJ, joint sounds, limitation of opening, gingival recession, inadequate occlusion, hearing disorders, headache, tenderness in the whole musculature of the stomatognathic system and cervical cancer. Objectives: To study the influence of low intensity laser therapy (LLI) in the treatment of temporomandibular disorders. Materials and methods: This is a systematic review. We consulted the following databases: BIREME, MEDLINE, PubMed, Science Direct and LILACS. The search terms used were "temporomandibular laser therapy" and "ATM laser treatment", in English and Portuguese. No search limits were established. During the search conducted by two independent reviewers, 1.344 articles were found, and after application of the exclusion criteria, 12 articles were selected for analysis. Results: Out of the 12 selected articles, only in one of them it was not observed any improvement in the painful TMJ because of the different types of TMJ. Conclusion: Despite the satisfactory results of laser use in reducing symptomatic pain in TMJ, the use of its parameters for each case is controversial, so we suggest that the foundation for other studies that these gaps may be filled with the larger goal of promoting a better quality of life for people who suffer any inconvenience in the TMJ region.

Keywords: Semiconductor lasers. Temporomandibular joint disorders. Physical therapy modalities.

\section{Introdução}

A disfunção temporomandibular (DTM) é definida como um termo coletivo que envolve um grande número de problemas clínicos que afetam os músculos mastigatórios, a articulação temporomandibular (ATM) e estruturas associadas, ou ambos (1). Dentre os principais sinais e sintomas das disfunções da ATM se encontram dores nos músculos da mastigação ou na ATM, ruídos articulares, limitação de abertura, retração gengival, oclusão inadequada, distúrbios auditivos, cefaleias, sensibilidade em toda musculatura do sistema estomatognático e cervical $(2,3,4)$.

0 sistema mastigatório é composto por vários componentes, e cada um apresenta uma tolerância estrutural específica. Os músculos mastigatórios (masseteres, temporais, pterigoideos laterais e mediais), ATM, dentes e estruturas de suporte dos mesmos são considerados estruturas de menor tolerância. Desse modo, a sensibilidade associada à dor nas estruturas supracitadas aumenta, resultando em limitação dos movimentos mandibulares devido à hiperatividade muscular, com inibição do fluxo sanguíneo normal aos tecidos, culminando em acúmulo de metabólitos nas células de tecidos musculares, o que proporciona um estado de espasmo, fadiga e dor no indivíduo $(1,5)$.

Por ser uma patologia complexa, a DTM tem originado diversos tipos de tratamento, sendo o mais eficaz, aquele desenvolvido em conjunto com uma equipe multidisciplinar (6). Os agentes físicos mais comumente associados ao seu tratamento são: termoterapia, eletroterapia, ultrassom, iontoforese, alguns agentes analgésicos e laser (7). Contudo, a laserterapia de baixa intensidade (LBI) tem demonstrado uma capacidade em auxiliar no tratamento sintomático da dor, promovendo um grau de conforto considerável ao paciente, momentos após sua aplicação (6). A laserterapia de baixa potência é uma radiação situada na porção visível do espectro das ondas eletromagnéticas, entre o infravermelho e o ultravioleta, e o comprimento de onda depende do tipo de substância estimulada $(8,9)$. É uma modalidade de tratamento não invasiva e de baixo custo, que vem sendo amplamente utilizada na prática clínica fisioterápica para o alívio de dor e regeneração tecidual. Dentre os efeitos terapêuticos, encontram-se: anti-inflamatório, 
analgésico, cicatrizante e modulador da atividade celular, os quais têm sido comprovados em diversos experimentos $(10,11)$. Dentre suas formas mais utilizadas, estão os lasers que utilizam o Arseneto de Gálio (AsGa) e o Arseneto de Gálio e Alumínio (AsGaAl), que têm gerado resultados favoráveis na terapêutica dos distúrbios musculoesqueléticos devido ao fato de seu poder de penetração ser relativamente alto, atingindo estruturas mais profundas (12).

Nesse contexto, objetivou-se estudar, por meio de uma revisão sistemática, a influência da laserterapia de diodo na reabilitação de portadores de DTM.

\section{Materiais e métodos}

Para a realização deste estudo, foram consultadas as bases de dados: BIREME, MEDLINE, PubMed, Science Direct e LILACS. Os termos de busca utilizados foram "temporomandibular laser therapy" e "ATM laser treatment", em inglês e português. Não foram estabelecidos limites de busca. Após uma pesquisa independente de dois revisores, os artigos foram contabilizados e o teste estatístico de Kappa foi utilizado para a verificação da concordância entre os dois pesquisadores $(K=0,629 ; p<0,0001)$. Quando havia dissenso na seleção dos estudos, um terceiro pesquisador era recrutado para decidir sobre sua inclusão ou não. Após a busca, foram encontrados 1.344 resultados e, posteriormente à aplicação dos critérios de exclusão, 12 artigos foram incluídos para o estudo. Foram incluídos os estudos experimentais e ensaios clínicos randomizados envolvendo seres humanos que apresentassem alguma das modalidades de intervenção da LBI nos DTM. Aqueles que não se enquadraram nesses critérios foram excluídos. Outra forma de exclusão se deu por meio da avaliação da qualidade metodológica dos estudos selecionados previamente pelos autores, bem como outras formas de uso da LBI que não a do AsGaAl e AsGa. Os artigos que foram apresentados em mais de uma base de dados foram contabilizados apenas uma vez.

\section{Resultados}

Pinheiro et al. (13) analisaram os efeitos da terapia LBI no tratamento de desordens maxilo-faciais em 165 indivíduos, sendo 141 do sexo feminino e 24 do sexo masculino. Foram tratados com o laser diodo seguindo os comprimentos de onda: $632,8 \mathrm{~nm}, 670$ $\mathrm{nm}$ e $830 \mathrm{~nm}$. As queixas mais relatadas foram: dor nas articulações temporomandibulares, neuralgia do trigêmeo, dor muscular e hipersensibilidade dentária tanto no pós-operatório quanto em hemangiomas pequenos. A maioria dos tratamentos consistiu de uma série de 12 aplicações (duas vezes por semana) e, em oito casos, uma segunda série foi aplicada. Os pacientes foram tratados com uma dose média de 2,5 $\mathrm{J} / \mathrm{cm}^{2}$. Ao fim do tratamento, 120 pacientes eram assintomáticos, 25 melhoraram consideravelmente e 20 permaneciam sintomáticos. Esses resultados indicaram que a LBI é uma ferramenta importante e traz muitos benefícios para o tratamento de muitas desordens da região maxilo-facial. Reduções nos níveis de dor também foram encontradas em outros estudos (14) que distribuíram, aleatoriamente, 18 pacientes do sexo feminino, com idade média de $27 \pm 7$ anos, com diagnóstico de DTM, em dois grupos: controle e tratado, utilizando a LBI nos seguintes parâmetros: $904 \mathrm{~nm}, 6 \mathrm{~J} / \mathrm{cm}^{2}, 0,38 \mathrm{~mW} / \mathrm{cm}^{2}$, área do feixe de 0,039 $\mathrm{cm}^{2}$, com modo de emissão contínua para o grupo tratado, enquanto no grupo controle foi realizada a mesma técnica de aplicação com o aparelho desligado.

Kulekcioglu et al. (15) fizeram um estudo com 35 pacientes para avaliar a efetividade do LBI como terapia nos DTM. Os pacientes foram divididos em grupo experimental $(n=20)$ e grupo controle $(n=$ 15). Além de um programa de exercício diário com padrão de amplitude de movimento, exercícios de alongamento e treinamento postural, todos os pacientes foram tratados com 15 sessões de laserterapia (comprimento de onda: $904 \mathrm{~nm}$; potência média de saída: $17 \mathrm{~mW}$; frequência: $1.000 \mathrm{~Hz}$; duração: 180 segundos; dosagem: $3 \mathrm{~J} / \mathrm{cm}^{2}$ ), sendo aplicadas para os quatro pontos mais dolorosos selecionados durante o exame. No grupo controle, o aparelho permanecia desligado. Como resultado, observou-se uma significativa redução da dor, além da melhora nos movimentos de abertura máxima, na lateralidade e no número de pontos hipersensíveis no grupo em que houve o tratamento real. A LBI foi, então, considerada pelos autores uma boa alternativa física para o tratamento de DTM. Em outro estudo (16), também foram evidenciadas melhoras na abertura bucal, com diminuição da sintomatologia dolorosa e dificuldades de mastigação de pacientes portadores de DTM.

Os efeitos do LBI também foram testados sobre a força da contração do músculo masseter em 15 pacientes com desconforto neuromuscular (dor) 
durante o fechamento, contra qualquer resistência, da ATM. A idade da amostra variou entre 19-29 anos, e a caneta do laser foi aplicada de forma pontual a uma distância de $2 \mathrm{~mm}$ da pele. Todos os pacientes apresentaram melhora na força de contração muscular, de cerca de 2,5 kgf para 3,01 kgf. Estes resultados sugerem que a aplicação do LBI é uma ferramenta eficaz para o tratamento de pacientes com dor orofacial que limitam a força de fechamento da ATM (17).

Outro estudo avaliou a eficiência imediata da LBP em 13 pacientes portadores de dor e disfunção da ATM. O lado mais comprometido pela DTM foi tratado com aplicação do laser, de comprimento de onda de $790 \mathrm{~nm}$, irradiado em quatro pontos na disfunção da ATM, com $1,5 \mathrm{~J} / \mathrm{cm}^{2}$; um ponto na região do ouvido externo, com $2,5 \mathrm{~J} / \mathrm{cm}^{2}$; em três pontos no músculo masseter, com $3 \mathrm{~J} / \mathrm{cm}^{2}$; e três pontos no músculo temporal, com $3 \mathrm{~J} / \mathrm{cm}^{2}$. Os músculos masseter e temporal, de ambos os lados, foram avaliados eletromiograficamente antes da LBI, imediatamente a aplicação do laser, 5 e 20 minutos após. Foram observadas reduções das atividades eletromiográficas medidas para todos os tempos, em ambos os músculos masseter e temporal (18).

León et al. (19) procuraram avaliar a eficácia da LBI nos DTM e, para isso, selecionaram 40 pacientes distribuídos, aleatoriamente, em dois grupos: 0 grupo A utilizou a terapia farmacológica (anti-inflamatórios e relaxante muscular), enquanto o grupo B recebeu a terapia laser em pontos de acupuntura: vesícula biliar 2 (vb2), estômago 7 (E7), estômago 6 (E6), na forma de aplicação pontual por 30 segundos/ponto e uma dose de $0,9 \mathrm{~J} / \mathrm{cm}^{2}$ por ponto. Imediatamente depois foi realizada uma aplicação, na forma de varredura, sobre as ATM afetadas numa área de $1 \mathrm{~cm}^{2}$ por cinco minutos. Constatou-se que a terapia a laser teve um resultado mais significativo quanto à resolução da dor em comparação com o grupo que utilizou a terapia farmacológica. Em outro estudo (20), vinte pacientes com DTM foram divididos aleatoriamente em dois grupos: (G1) com média de idade igual a 28,2 \pm 7 anos, tratados com técnicas de terapia manual, e (G2), com média de idade igual a $24,01 \pm 6,04$, tratados com a associação de técnicas de terapia manual e LBI: $904 \mathrm{~nm}, 6 \mathrm{~J} / \mathrm{cm} 2,0,38 \mathrm{~mW} / \mathrm{cm}^{2}$. Observou-se a redução significativa do nível de dor em ambos os grupos tratados, porém no (G2), a redução da sintomatologia dolorosa foi mais relevante.

Utilizando a LBI de $660 \mathrm{~nm}$ e $890 \mathrm{~nm}$ na redução da dor nos músculos mastigatórios por meio de um estudo duplo-cego e controlado com placebo, 16 pacientes foram divididos aleatoriamente em dois grupos. Para o grupo laser, duas sondas de laser $(660 \mathrm{~nm}$, $6,2 \mathrm{~J} / \mathrm{cm}^{2}, 6 \mathrm{~min}$, onda contínua, e $890 \mathrm{~nm}, 1 \mathrm{~J} / \mathrm{cm}^{2}$, $10 \mathrm{~min}, 1500 \mathrm{~Hz}$ ) foram utilizadas nos músculos dolorosos. Para o grupo controle, o tratamento foi similar, mas os pacientes não foram irradiados. 0 tratamento foi administrado duas vezes por semana durante três semanas. Em ambos os grupos experimentais, a redução da dor após o tratamento foi estatisticamente significativa, sendo o laser com menor comprimento de onda mais efetivo para a redução da dor (21).

Venezian et al. (22) avaliaram o efeito do laser de diodo $(780 \mathrm{~nm})$ na dor à palpação e eletromiográficos (EMG) dos músculos masseter e temporal anterior. $\mathrm{O}$ laser foi aplicado em músculos temporal e masseter duas vezes por semana, por quatro semanas. Quarenta e oito pacientes com dor miofascial foram distribuídos aleatoriamente entre tratamento experimental e controle e entre doses energéticas de $25 \mathrm{~J} / \mathrm{cm}^{2}$ e $60 \mathrm{~J} / \mathrm{cm}^{2}$, foram avaliados utilizando a escala numérica de dor antes e imediatamente após a aplicação final, e 30 dias após o tratamento com laser. A eletromiografia de superfície foi realizada antes e após a terapia a laser. Os resultados mostraram que houve diferença estatisticamente significativa na atividade EMG entre os grupos, antes e após o tratamento com laser. No que diz respeito à dor à palpação, embora ambos os grupos apresentassem uma diferença significativa nos sintomas antes e após o tratamento, somente os indivíduos do grupo experimental demonstraram reduções estatisticamente significativas no nível de dor em todas as regiões dos músculos palpados. No entanto, não houve diferença estatisticamente significativa entre os grupos (experimental e placebo). Em conclusão, o LBI não promoveu qualquer alteração na atividade EMG. 0 tratamento, porém, diminuiu os sintomas da dor no grupo experimental.

Conti (23) avaliou a eficácia da LBI em 20 pacientes, todos com queixa principal de dor miogênica e artrogênica na ATM. Para isso, foi realizado o tratamento a laser com comprimento de onda de 830 $\mathrm{nm}$ e potência de $4 \mathrm{~J} / \mathrm{cm}^{2}$. Concluiu que houve uma melhora na dor relatada apenas para os pacientes com dor miogênica, enquanto para os pacientes com dor artrogênica o tratamento a laser resultou em uma discreta melhora na abertura vertical total da ATM, sem diferenças estatisticamente significativas para a redução da dor. Nos estudos de Venâncio et al. 
(24), a eficácia da LBI foi verificada em 30 pacientes portadores de DTM, por meio de um estudo duplo-cego. A amostra foi dividida em dois grupos: (1) grupo experimental e (2) grupo placebo. 0 primeiro foi submetido ao tratamento com laser infravermelho seguindo os parâmetros:

$780 \mathrm{~nm}, 30 \mathrm{~mW}, 10 \mathrm{~s}, 6,3 \mathrm{~J} / \mathrm{cm}^{2}$ em três pontos da ATM. Concluiu-se que não houve diferença significativa entre ambos os grupos.

\section{Discussão}

A radiação laser de baixa potência tem sido cada vez mais empregada nas condutas de reabilitação dos DTM (14). Esta tem sido empregada como um agente biomodulador capaz de promover efeitos analgésicos e anti-inflamatórios por meio de induções de respostas celulares e sistêmicas $(25,26)$. 0 sucesso do tratamento de patologias com o laser na região maxilo-facial tem sido demonstrado, e seu uso ocorre em razão dos efeitos analgésicos explicados pelo aumento dos níveis de beta-endorfinas, aumento do limiar de descarga de dor, diminuição de bradicinina e liberação de histamina, aumento do fluxo linfático, diminuição do edema e substâncias álgicas, aumento do fornecimento do sangue, redução do tempo de inflamação e promoção do relaxamento muscular (27-31).

O sintoma prevalente no portador de DTM é a dor, podendo, ainda, estar isolada ou mesmo associada a outras queixas, como estalidos articulares, tensão muscular, desgaste/fratura de dentes e rigidez articular. Nesse contexto, o laser de baixa intensidade, segundo Almeida (32), vem sendo empregado como um meio físico no tratamento das DTMs, em função de seus efeitos terapêuticos. No entanto, apesar de serem utilizados para tratamento de várias condições (33), os efeitos dependem da dosimetria e de condições corporais sistêmicas (34).

Pode-se observar que, na maioria dos estudos citados, apesar dos diversificados parâmetros de aplicação da laserterapia, houve reduções dos sintomas dos portadores de DTM, sobretudo na dor, o que, para Okenson (1), constitui elemento-chave na reabilitação da ATM acometida, visto que a dor é um agente limitante para os movimentos normais da ATM, bem como o sintoma mais relatado pelos acometidos. Os efeitos analgésicos induzidos pela laserterapia podem ser justificados por meio da modulação dos me- diadores químicos da inflamação, além do estímulo à síntese de beta endorfina. Esses fatores associados tendem a limitar a redução do limiar de excitabilidade dos receptores dolorosos (35) e eliminar substâncias alogênicas, por meio da modulação do metabolismo celular basal induzida pela endorfina que, em última instância, promoverá uma ação anti-inflamatória (36-39). Uma possível explicação para a redução dos espasmos na musculatura da ATM tratada com laserterapia (40) pode estar associada aos níveis de energia absorvida nos citocromos e porfirinas dentro da mitocôndria e membrana celular dessas células. Tais níveis de energia disponibilizarão uma pequena quantidade de oxigênio extra dentro da célula, acelerando a glicólise e a oxidação fosforilativa com maior disponibilização de ATP, além da ativação da microcirculação que, pela ação da histamina liberada por meio da ação do próprio laser, proporcionará mais nutrientes para que essas células possam otimizar seus metabolismos (41).

A resposta do paciente não dependerá somente do tipo de laser, mas também do tecido alvo em questão, bem como das condições imunológicas. Contudo, sabe-se que na maioria dos casos, o melhor resultado é atingido quando as terapias estão associadas, e a laserterapia pode contribuir satisfatoriamente.

\section{Conclusão}

A LBI tem se mostrado uma alternativa importante no alívio da dor e no restabelecimento da função, no que diz respeito ao tratamento das DTM. Por ser uma modalidade de tratamento não invasiva e de baixo custo, vem sendo frequentemente utilizada na prática clínica.

Apesar das poucas evidências clínicas, o LBI de AsGaAl e AsGa mostraram ser eficazes no tratamento dos DTM na maioria dos estudos descritos. Todavia, a falta de consenso sobre os parâmetros utilizados é evidente e parece ser um fator limitante para conclusões mais acuradas a respeito do nível da eficácia do uso da LBI nesses casos. Os autores sugerem que novos estudos sejam realizados, de preferência do tipo ensaios clínicos randomizados, e que parâmetros específicos sejam estudados e seus efeitos divulgados junto à comunidade científica e clínica a fim de otimizar os efeitos da LBI, sobretudo para que os pacientes que sofrem algum tipo de DTM possam se beneficiar, melhorando sua qualidade de vida. 


\section{Referências}

1. Okenson JP. Fundamentos de oclusão e desordens temporomandibulares. São Paulo: Artes Médicas; 2000.

2. Favero K. Disfunções da articulação temporomandibular: uma visão etiológica e terapêutica multidisciplinar [dissertação]. São Paulo: Centro de Especialização em Fonoaudiologia Clínica Motricidade Oral; 1999.

3. Molina O, Mazzetto M, Stechman J, Santos JR, Fernandes $\mathrm{R}$, Pizzo R, et al. Distúrbios internos articulares. Retrodiscite em pacientes com Bruxismo e DCM-Características clínicas, diagnóstico e sugestões para tratamento. J Bras Oclusão, ATM e Dor Orofacial. 2001; 1(1):67-75.

4. Costa LF, Guimarães JP, Chaobas A. Prevalência de distúrbios da articulação temporomandibular em crianças e adolescentes brasileiros e sua relação com má-oclusão e hábitos parafuncionais: um estudo epidemiológico transversal. J Bras Ortodontia \& Ortopedia Facial. 2004;9(50):162-9.

5. Siqueira JTT, Teixeira MJ. Dor músculo-esquelética do segmento cefálico. Rev Med. 2001;80(2):290-6.

6. Carrasco TG, Mazzetto MO, Mazzetto RG, Mestriner W. Low intensity laser therapy in temporomandibular disorder: a phase II double-blind study. Cranio. 2008; 26(4):274-81.

7. Carvalho CM, Lacerda JA, Santos FP, Cangussu MC, Marques AM, Pinheiro AL. Wavelength effect on temporomandibular joint pain: a clinical experience. Lasers Med Sci. 2010;25(2):229-32.

8. Assunção D, Stall KR, Castilho LV, Amorim MH, Palma M, Fonseca PB. Tratamento fisioterapêutico da acne por meio do laser. Fisioter Mov. 2003;16(4):11-6.

9. Low J, Reed A. Eletroterapia explicada: princípios e prática. São Paulo: Manole; 2001.

10. Kato MT, Kogawa EM, Santos CN, Conti PCR. Tens and low-level laser therapy in the management of temporomandibular disorders. J Appl Oral Sci. 2006; 14(2):130-5.

11. Fikackova H, Dostalova L, Vosicka R, Peterova V, Navratil $\mathrm{L}$, Lesak J. Arthralgia of the temporomandibular joint and low-level laser therapy. Photomed Laser Surg. 2006;24(4):522-7.
12. Genovese WJ. Revisão laser. São Paulo: Pancast; 2000.

13. Pinheiro AL, Cavalcanti ET, Pinheiro TI, Alves MJ, Manzi CT. Low-level laser therapy in the management of disorders of the maxillofacial region. J Clin Laser Med Surg. 1997;15(4):181-3.

14. Frare JC, Nicolau RA. Análise clínica do efeito da fotobiomodulação laser (GaAs - $904 \mathrm{~nm}$ ) sobre a disfunção temporomandibular. Rev Bras Fisioter. 2008; 12(1):37-42.

15. Kulekcioglu S, Sivrioglu K, Ozcan O, Parlak M. Effectiveness of low-level laser therapy in temporomandibular disorder. Scand J Rheumatol. 2003;32(2):114-8.

16. Cetiner S, Kahraman KS, Yuçetas S. Evaluation of lowlevel laser therapy in the treatment of temporomandibular disorders. Photomed Laser Surg. 2006;24(5): 637-41.

17. Medeiros JS, Vieira GF, Nishimura PY. Laser application effects on the bite strength of the masseter muscle, as an orofacial pain treatment. Photomed Laser Surg. 2005;23(4):373-6.

18. Shinozaki EB, Paiva G, Zanin FAA, Brugnera A. Avaliação eletromiográfica de pacientes com DTM após a laserterapia. RGO. 2006;54(4):334-9.

19. León IG, Almagro S, Garcia RC. Los trastornos temporomandibulares y la radiación láser. Rev Cubana Estomatol. 2007;44(3):1-8.

20. Andrade TN. Estudo comparativo entre os efeitos de técnicas de terapia manual isoladas e associadas à laserterapia de baixa potência sobre a dor em pacientes com disfunção temporomandibular. RGO. 2008; 56(3):287-95.

21. Shirani AM, Gutknecht N, Taghizadeh M, Mir M. Lowlevel laser therapy and myofacial pain dysfunction syndrome: a randomized controlled clinical trial. Lasers Med Sci. 2009;24(5):715-20.

22. Venezian GC, Silva MA, Mazzetto RG, Mazzetto MO. Low level laser effects on pain to palpation and electromyographic activity in TMD patients: a doubleblind, randomized, placebo-controlled study. Cranio. 2010;28(2):84-91.

23. Conti PC. Low level laser therapy in the treatment of temporomandibular disorders (TMD): a double-blind pilot study. Cranio. 1997;15(2):144-9. 
24. Venâncio RA, Camparis CM, Lizarelli RF. Low intensity laser therapy in the treatment of temporomandibular disorders: a double-blind study. J Oral Rehabil. 2005; 32(11):800-7.

25. Mazzetto MO, Carrasco TG, Bidinelo EF, Pizzo RCA, Mazzetto RG. Low intensity laser application in temporomandibular disorders: a phase I double-blind study. Cranio. 2007;25(3):186-92.

26. Mazzetto MO, Hotta TH, Pizzo RCA. Measurements of Jaw Movements and TMJ Pain Intensity in Patients Treated with GaAlAs Laser. Braz Dent J. 2010;21(4): 356-60.

27. Kobayashi M, Kubota J. Treatment of temporomandibular joint (TMJ) pain with diode laser therapy. Laser Therapy. 1999;11(1):11-8.

28. Kato MT, Kogawa EM, Santos CN, Conti PCR. Tens and low-level laser therapy in the management of temporomandibular disorders. J Appl Oral Sci. 2006; $14(2): 130-5$.

29. Núñez SH, Garcez AS, Suzuki SS, Ribeiro MS. Management of mouth opening in patients with temporomandibular disorders through low-level laser therapy and transcutaneous electrical neural stimulation. Photomed Laser Surg. 2006;24(1):45-9.

30. Cunha LA, Firoozmand LM, Silva AP, Esteves SA, Oliveira W. Efficacy of low-level laser therapy in the treatment of temporomandibular disorder. Int Dent J. 2008;58(4):213-7.

31. Fikackova H, Dostalova T, Navratil L, Klaschka J. Effectiveness of low-level laser therapy in temporomandibular joint disorders: a placebo-controlled study. Photomed Laser Surg. 2007;25(4):297-303.

32. Almeida L. Laserterapia na Odontologia. Biodonto. 2004;1:11-53.

33. Hansen HJ, Thoroe U. Low power laser biostimulation of chronic oro-facial pain. A double-blind placebo controlled cross-over study in 40 patients. Pain. 1990; 43(2):169-79.
34. Kubota J, Ohshiro T. The effects of diode laser low reactive level laser therapy (LLLT) on flap survival in a rat model. Laser Therapy. 1989;1(3):127-35.

35. Campana EA, Moya M, Gavotto A, Juri H, Palma JA. The relative effects of He Ne laser and meloxicam on experimentally inced inflammation. Laser Therapy. 1999;11(1):36-41.

36. Chavantes MC, Janete AD. Aplicação de laser na área cardiovascular. Arq Bras Cardiol. 1990;54(1):63-8.

37. Simunovic Z, Trobonjaca T, Trobonjaca Z. Treatment of medial and lateralepicondylitis - tennis and golfer's elbow - with low level laser therapy: a multicenter double blind, placebo controlled clinical study on 324 patients. J Clin Laser Med Surg. 1998;16(3):145-51.

38. Wilden L, Karthein R. Import of radiation phenomena of electrons and therapeutic low-level-laser in regard to the mitochondrial energy transfer. J Clin Laser Med Surg. 1998;16(3):159-65.

39. Karu T. Primary and secondary mechanisms of action of visible-to-near IR radiation on cells. J Photochem Photobiol B. 1999;49(1):1-17.

40. Schindl A, Schindl M, Pernerstorfer-Schon H, Schindl L. Low-intensity laser therapy: a review. J Investig Med. 2000;48(5):312-26.

41. Karu TI. Molecular mechanism of the therapeutic effect of low-intensity laser radiation. Dokl Akad Nauk SSSR. 1986;291(5):1245-9.

Recebido: 20/10/2011

Received: 10/20/2011

Aprovado: 21/03/2012

Approved: 03/21/2012 